\title{
Offset balancing in pseudo-correlation radiometers for CMB measurements
}

\author{
A. Mennella ${ }^{1}$, M. Bersanelli ${ }^{2}$, M. Seiffert ${ }^{3}$, D. Kettle ${ }^{4}$, N. Roddis ${ }^{4}$, A. Wilkinson ${ }^{4}$, and P. Meinhold ${ }^{5}$ \\ 1 IASF-CNR, Sezione di Milano, Via Bassini 15, 20133 Milan, Italy \\ 2 Università degli Studi di Milano, Via Celoria 16, 20133 Milan, Italy \\ 3 Jet Propulsion Laboratory, California Institute of Technology, Pasadena, CA 91109, USA \\ 4 Jodrell Bank Observatory, Jodrell Bank, Macclesfield, Cheshire SK11 9DL, UK \\ 5 University of California at Santa Barbara, Physics Department, Santa Barbara, CA 93106, USA
}

Received 26 March 2003 / Accepted 7 August 2003

\begin{abstract}
Radiometeric CMB measurements need to be highly stable and this stability is best obtained with differential receivers. The residual $1 / f$ noise in the differential output is strongly dependent on the radiometer input offset which can be cancelled using various balancing strategies. In this paper we discuss a software method implemented in the PLANCK-LFI pseudocorrelation receivers which uses a tunable gain modulation factor, $r$, in the sky-load difference. Numerical simulations and experimental data show how proper tuning of the parameter $r$ ensures a very stable differential output with knee frequencies of the order of few mHz. Various approaches to calculate $r$ using the radiometer total power data are discussed with some examples relevant to PLANCK-LFI. Although the paper focuses on pseudo-correlation receivers and the examples are relative to PLANCK-LFI, the proposed method and its analysis is general and can be applied to a large class of differential radiometric receivers.
\end{abstract}

Key words. cosmology: cosmic microwave background, observations - instrumentation: detectors - methods: analytical

\section{Introduction}

The dramatic progress achieved in the past decade in Cosmic Microwave Background (CMB) observations, particularly in anisotropy experiments, is strongly correlated with the remarkable improvements obtained in microwave and sub-millimetre detector technology, as well as in cryogenic technology (for a recent review see, e.g., Bersanelli et al. 2002). Single-detector sensitivities of $0.1-0.4 \mathrm{mK} \mathrm{Hz}^{-1 / 2}$ have been demonstrated for cryogenic operation of Indium Phosphide HEMT (High Electron Mobility Transistors) amplifiers (typically cooled at $\sim 20 \mathrm{~K}$ ) in the range $25-100 \mathrm{GHz}$. At higher frequencies, sensitivities at a level NEP $\sim 1 \times 10^{-17} \mathrm{~W} \times \mathrm{Hz}^{-1 / 2}$ have been achieved by spider web bolometers cooled to $\sim 0.1 \mathrm{~K}$ (see, e.g., Lamarre 1997). Moderate-size arrays of such detectors can today produce high resolution full-sky maps of the CMB with a high signal-to-noise ratio. These ultra-sensitive systems impose stability requirements that are proportionally stringent and call for highly optimised instrument design. In particular, the instrument needs to be immune at $\mu \mathrm{K}$ level from the effect of parasitic signals introduced by non-idealities in the system, which would propagate as systematic errors in the final CMB maps (Mennella et al. 2002).

Send offprint requests to: A. Mennella, e-mail: daniele@mi.iasf.cnr.it
In coherent radiometeric systems one of the major concerns is the intrinsic instability due to gain and noise temperature fluctuations of the amplifiers themselves, typically well represented by a $1 / f$-type noise spectrum. Differential receivers, such as the Dicke-switched scheme, reduce the impact of amplifier instabilities in the measured signal with a fast (typically $\sim 100 \mathrm{~Hz}$ ) switch between the sky input port and a stable reference, sometimes given by another horn pointed at the sky. Dicke-type receivers have a long history in CMB observations and were successfully employed in the COBE-DMR instrument that first detected CMB anisotropies (Smoot et al. 1990, 1992).

In recent years, a scheme called "pseudo-correlation" radiometer has been introduced to improve over the classical Dicke scheme. As it will be discussed in detail, this design has a two-port front-end that allows a continuous comparison between the sky signal $T_{\text {sky }}$ and a stable reference signal $T_{\text {ref }}$, improving the sensitivity by a factor $\sqrt{2}$ over a Dicke radiometer. In addition, fast (few $\mathrm{kHz}$ ) phase switching provides immunity from back-end fluctuations. Different versions of pseudocorrelation designs are being used for the second and third generation of space-based radiometric instruments for CMB anisotropy: NASA's Wilkinson Microwave Anisotropy Probe (WMAP) and the Low Frequency Instrument (LFI) on board ESA's Planck mission. 
In principle, a perfectly balanced pseudo-correlation radiometer is completely free from $1 / f$ effects. Residual sensitivity to $1 / f$ noise, as well as to other systematic effects, is proportional to the input offset $\Delta T_{\text {off }} \equiv T_{\text {ref }}-T_{\text {sky }}$ at the level of the first hybrid coupler in the radiometer front-end. In practice full balance is not achievable, so that a key instrument design objective is to minimise $\Delta T_{\text {off }}$.

In the case of the WMAP instrument, the radiometers directly measure temperature differences between sky signals from two widely separated regions of the sky (Jarosik et al. 2003; Bennett et al. 2003). This is accomplished with a symmetric back-to-back double-telescope system, and pairs of feeds which provide the two inputs to the pseudo-correlation front-end. In this scheme, the contribution to the offset from external signals is only of few $\mathrm{mK}$ (dominated by the CMB dipole or, at low frequencies, by the Galactic plane). The offset (of order $\Delta T_{\text {off }}<1 \mathrm{~K}$ ) is dominated by second-order instrument asymmetries.

The LFI radiometers, instead, measure differences between the sky, $T_{\text {sky }}$, and a stable internal cryogenic reference load $\left(T_{\text {ref }}\right)$ cooled at about $4 \mathrm{~K}$ by the pre-cooling stage of the High Frequency Instrument (HFI) bolometer array in the Planck focal plane. This introduces an offset of order $\Delta T_{\text {off }} \sim 2$ to $3 \mathrm{~K}$. The effects of this offset, however, are compensated by introducing a "gain modulation factor", $r$, which balances the output in the on-board signal processing (Bersanelli et al. 1995; Seiffert et al. 2002). Experimental results from advanced LFI prototypes (Meinhold et al. 1998; Tuovinen et al. 2000), as well as analytical calculations, show that great immunity from $1 / f$ effects can be obtained when the value of $r$ is accurately selected.

Two different approaches can be used to select the value of $r$ : it can either be controlled in hardware by adjusting (in principle in real-time) a variable gain to achieve the null-output condition; or it can be set as a controllable software parameter, giving more flexibility to the system. The latter approach was adopted for the LFI.

As we shall see in more detail, the best estimate of $r$ can be obtained based on the radiometer data themselves, collected in a raw, undifferenced form. In a space application this may impose non-trivial demands on the telemetry rate. If the data available to calculate $r$ are limited by telemetry constraints, this may limit the accuracy of the determination of $r$ and therefore limit the instrument stability.

A detailed analytical study of the impact of non-idealities in the LFI radiometer, in particular $1 / f$ noise effects, has been carried out in a previous work (Seiffert et al. 2002). In this paper we discuss in detail the required and obtainable accuracy of $r$, and its effect on the measurement quality. Although we focus on offset balancing in pseudo-correlation radiometers and discuss some examples in the context of PLANCK-LFI, the concepts and formalism discussed in our paper are general and applicable to any switched radiometer like unbalanced Dicke receivers.

After a short description of the radiometer concept, we discuss the issue of offset balancing and derive the optimal $r$ for $1 / f$ noise minimisation (Sect. 3), demonstrating its effectiveness by applying it to representative simulated data streams.
In addition we discuss the impact of the choice of $r$ on other systematic effects and derive the required accuracy on the calculation of $r$. In Sect. 4 we present and compare various approaches for calculating $r$ and show how these can be used in the context of space experiments; an example of the application of this offset-balancing technique to laboratory radiometer data is presented and discussed in Sect. 5. Finally, in Sect. 6 we discuss the impact of systematic effects of instrumental and astrophysical origin on the calculation of $r$.

\section{Pseudo-correlation differential radiometers: Basic concepts}

The Dicke switched radiometer represents a typical implementation of a differential receiver. In its simplest form a switch located in the front-end commutes rapidly between the sky and the reference horns so that a sequence of sky-load signals is detected and differenced at the output of the amplification chain; the radiometer sensitivity per unit integration time is given by:

$\Delta T_{\mathrm{rms}}=2 \frac{T_{\mathrm{sys}}}{\sqrt{\beta}}$

where $T_{\text {sys }}=T_{\text {sky }}+T_{\text {noise }}$ is the system temperature, $T_{\text {noise }}$ represents the radiometer noise temperature and $\beta$ is the bandwidth.

If the switching frequency is sufficiently high so that the gain can be considered nearly constant in a sky-load cycle, then the susceptibility to gain fluctuations is very much improved:

$\frac{\Delta T_{\mathrm{Gain}}}{T_{\mathrm{sys}}}=\frac{\Delta G}{G} \frac{T_{\mathrm{sky}}-T_{\mathrm{ref}}}{T_{\mathrm{sys}}}$,

where $\Delta T_{\text {Gain }}$ represents the rms signal variation caused by gain fluctuations and $T_{\text {sky }}, T_{\text {ref }}$ represent the sky and reference load temperatures, respectively.

Although this scheme is effective in reducing $1 / f$ noise in the final measurements, the presence of a lossy active component in the front-end increases the radiometer noise and may introduce additional $1 / f$ components. These limitations, however, can be overcome with a modified version of the Dicke scheme, the pseudo-correlation radiometer, that has been recently adopted for the WMAP and PLANCK-LFI instruments.

In Fig. 1 we show a schematic of a pseudo-correlation radiometer in its simplest form. The sky and reference signals are summed by a front-end $180^{\circ}$ hybrid, amplified by two parallel amplification chains and then separated by a second hybrid, detected and differenced. In this way the $1 / f$ noise from the RF amplification stages is the same in both signals, so that they can be eliminated at first order by differencing.

To reduce the effect of instabilities in the back-end electronics a fast switching between the two inputs is implemented in the two radiometer legs; as an example of application of the switched pseudo-correlation scheme, Fig. 2 shows a schematic of the radiometers used in the PLANCK-LFI instrument.

In the front-end part (see top part of figure) the radiation entering the feed-horn is separated by an OrthoMode Transducer (OMT) into two perpendicular linearly polarised components that propagate independently through two parallel radiometers. 


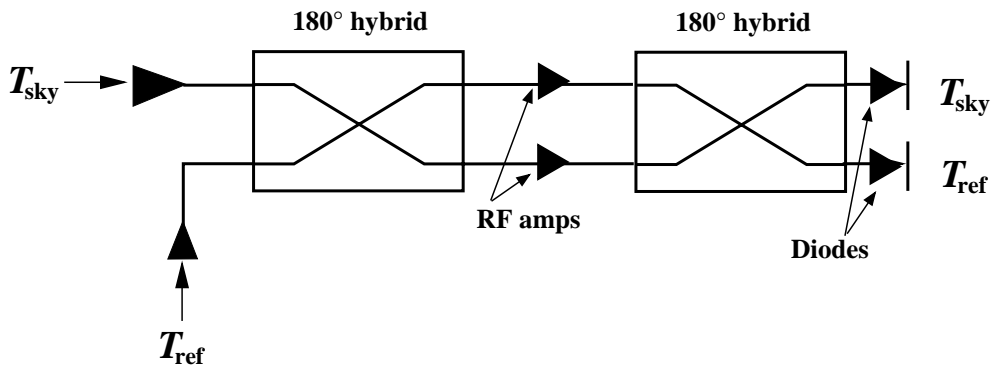

Fig. 1. A pseudo-correlation radiometer in its simplest form. This scheme allows a differential measurement without requiring an active switch before the first $\mathrm{RF}$ gain stage.
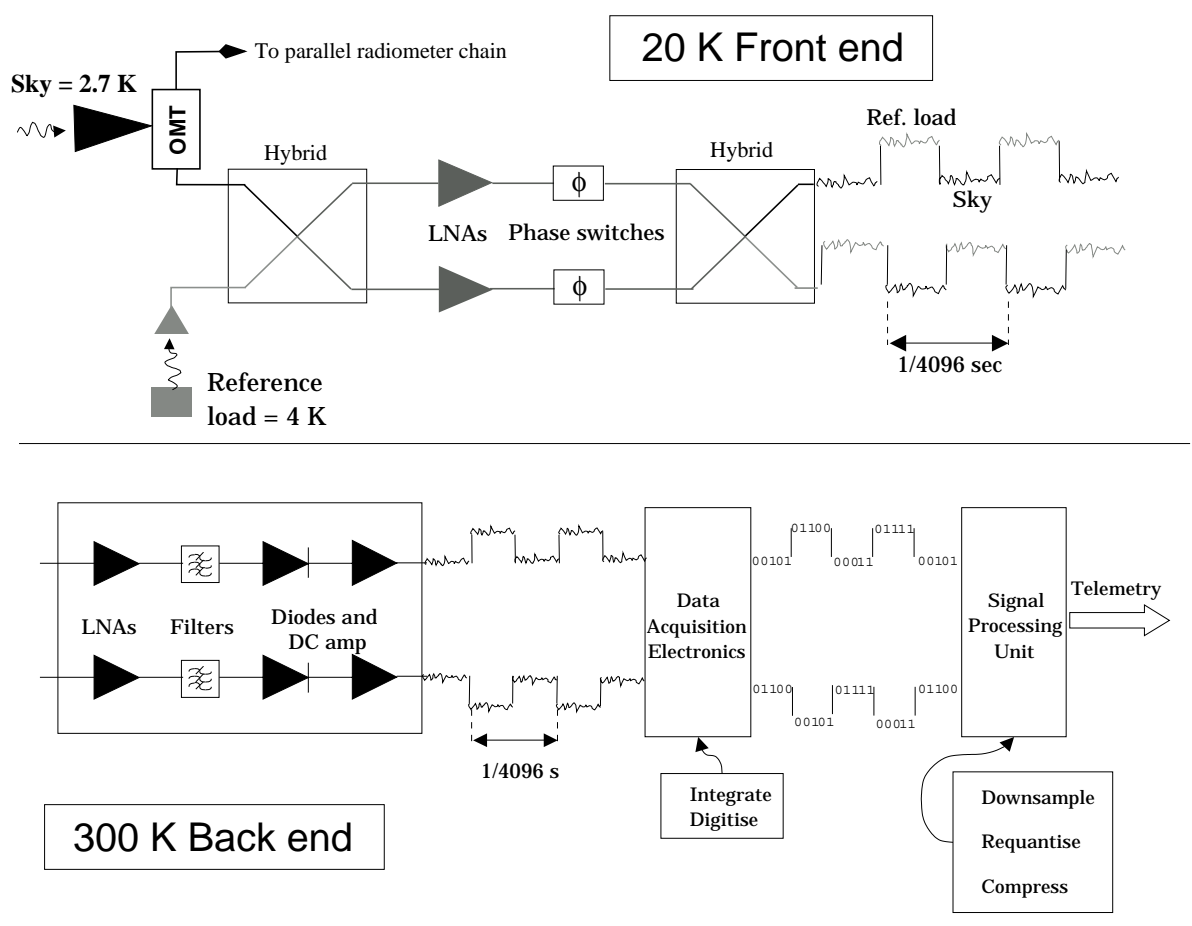

Fig. 2. Baseline LFI pseudo-correlation radiometer. One of the two $180^{\circ}$ phase switches in the front end switches rapidly the phase of the propagating signal, thus producing a sequence of sky-load signals at the output of the second hybrid. In the warm back-end the signal are further amplified, detected and digitised before telemetry.

In each radiometer, the sky signal and the signal from a stable reference load at $\sim 4 \mathrm{~K}$ are coupled to cryogenic low-noise HEMT amplifiers via a $180^{\circ}$ hybrid. One of the two signals then runs through a switch that applies a phase shift which oscillates between 0 and $180^{\circ}$ at a frequency of $4096 \mathrm{~Hz}^{1}$ (the second phase switch is present for symmetry on the second radiometer leg but it does not introduce any phase shift). The signals are then recombined by a second $180^{\circ}$ hybrid coupler, producing a sequence of sky-load signals at the output alternating at twice the frequency of the phase switch.

In the back-end of each radiometer (see bottom part of Fig. 2) the RF signals are further amplified, filtered by a lowpass filter and then detected. After detection the sky and reference load signals are integrated and digitised before sending to ground. Note that the current PLANCK-LFI available telemetry allows the downloading of the total power data streams for all the detectors, so that the sky-load differecing will be performed "off-line" during ground data analysis.

\footnotetext{
1 This frequency corresponds to a full $0-180^{\circ}$ cycle, so that the rate between two subsequent switch states is $8192 \mathrm{~Hz}$.
}

\section{Offset balancing and $1 / f$ noise minimisation}

In differential radiometers it is desirable to maintain the offset between the sky and reference signals as small as possible in order to minimise the effect of gain instabilities (see Eq. (2)). An offset introduced by an internal reference load can be balanced before differencing either by a variable back-end gain stage with a feed-back scheme to maintain the power output as close as possible to zero, or by by multiplying in software one of the two signals by a so-called gain modulation factor. In both cases the differential radiometer output can be written as:

$$
\begin{aligned}
& p_{\text {out }}=a G_{\text {tot }} k_{\mathrm{B}} \beta\left[\tilde{T}_{\text {sky }}+T_{\mathrm{n}}-r\left(\tilde{T}_{\text {ref }}+T_{\mathrm{n}}\right)\right] \\
& \tilde{T}_{\text {sky }}=T_{\text {sky }} / L_{\text {sky }}+\left(1-L_{\text {sky }}^{-1}\right) T_{\text {phys }} \\
& \tilde{T}_{\text {ref }}=T_{\text {ref }} / L_{\text {ref }}+\left(1-L_{\text {ref }}^{-1}\right) T_{\text {phys }}
\end{aligned}
$$

where $L_{\text {sky }}$ and $L_{\text {ref }}$ are the insertion losses of the front-end sky and reference load antennas, $T_{\text {phys }}$ is the physical temperature of the receiver front-end, $a$ is the detector proportionality constant, $G_{\text {tot }}$ the radiometer total gain, $k_{\mathrm{B}}$ the Boltzmann constant, 
$\beta$ the radiometer bandwidth and $r$ the gain modulation factor. From Eq. (3) it follows that $p_{\text {out }}=0$ for $r=r_{0}^{*}$ where

$r_{0}^{*}=\frac{\tilde{T}_{\text {sky }}+T_{\mathrm{n}}}{\tilde{T}_{\text {ref }}+T_{\mathrm{n}}}$.

In the next section we will show how the above condition of zero power output represents a very good approximation of a balanced radiometer and is very effective in reducing the radiometer susceptibility to gain fluctuations to very low levels.

In the case of PLANCK-LFI the radiometer offset has been balanced through a software scheme that uses the undifferenced total power data to calculate $r$, which is expected to be constant on timescales as long as several days. This scheme has been preferred to a hardware gain modulation because it simplifies the radiometer hardware and avoids potential systematic errors from the variable gain stage.

\subsection{Analytical derivation of the balancing condition}

In this section we calculate the value of the gain modulation factor that cancels the radiometer susceptibility to front-end gain fluctuations and show that this value is very well approximated by the the value $r_{0}^{*}$ in Eq. (4).

Before proceeding into the details of the calculation we briefly examine the expected magnitude of gain and noise temperature fluctuations in the total power noise streams; more details concerning the analytical treatment of LFI pseudocorrelation radiometers can be found in Seiffert et al. (2002).

\subsubsection{Noise fluctuations in total power streams}

Cryogenic HEMT amplifiers are known to have $1 / f$ fluctuations in gain and noise temperature (Pospieszalski 1989; Wollack 1995; Jarosik 1996). The level of these fluctuations can vary considerably among amplifiers and depends on the details of device fabrication, device size, circuit design, and other factors. Because of this, we adopt an empirical model for the fluctuations also described in Seiffert et al. (2002).

In particular we write the $1 / f$ spectrum of the gain fluctuations as

$\frac{\Delta G(f)}{G}=\frac{C}{f^{\alpha}}$,

where $0.5 \lesssim \alpha \lesssim 1$ and $C$ represents a constant normalization factor. Similarly, we can write the noise temperature fluctuations as

$\frac{\Delta T_{\mathrm{n}}(f)}{T_{\mathrm{n}}}=\frac{A}{f^{\alpha}}$,

where $A \sim C /\left(2 N_{\mathrm{s}}\right)$ is the normalization constant for noise temperature fluctuations. In the case of the PLANCK-LFI radiometers we use the estimates $A \simeq 1.8 \times 10^{-5}$ for the 30 and $44 \mathrm{GHz}$ and $A \simeq 2.5 \times 10^{-5}$ for the 70 and $100 \mathrm{GHz}$ radiometers.

\subsubsection{Derivation of the optimal gain modulation factor for $1 / f$ minimisation}

Following the approach described in Seiffert et al. (2002) it is possible to derive analytical formulas for the knee frequency

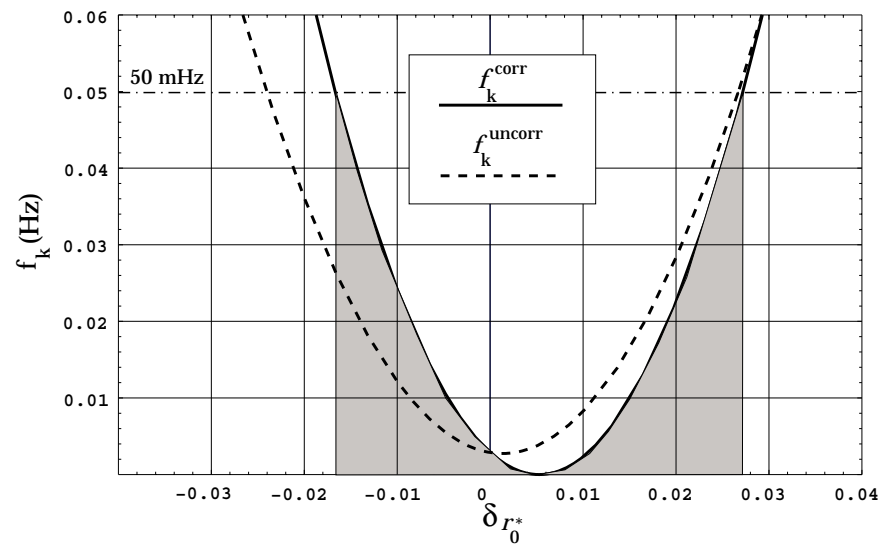

Fig. 3. Radiometer knee frequency versus the relative accuracy $\delta r / r=$ $\left(r-r_{0}^{*}\right) / r_{0}^{*}$ for the $70 \mathrm{GHz}$ PLANCK-LFI channel. The solid and dashed curves represent the values of the knee frequency calculated assuming correlated and uncorrelated fluctuations in the front-end amplifiers. The dashed-dotted $50 \mathrm{mHz}$ line indicates the PLANCK-LFI design value for the knee frequency, while the shaded area represents the range of accuracy that is compliant with this design value.

relative to $1 / f$ fluctuations of the differenced data streams induced by front-end gain and noise temperature fluctuations.

If we assume that gain and noise temperature fluctuations are correlated then we obtain the following relationship for the knee frequency:

$$
\begin{aligned}
f_{\mathrm{k}}^{\mathrm{corr}}= & \left\{\frac { C } { \tilde { T } _ { \mathrm { sky } } + T _ { \mathrm { n } } } \sqrt { \frac { \beta } { 2 } } \left[\left(\tilde{T}_{\text {sky }}+(1+A / C) T_{\mathrm{n}}\right)\right.\right. \\
& \left.\left.-r\left(\tilde{T}_{\text {ref }}+(1+A / C) T_{\mathrm{n}}\right)\right]\right\}^{1 / \alpha},
\end{aligned}
$$

which is zero for the following value of $r$ :

$r=r_{\text {corr }}^{*}=\frac{\tilde{T}_{\text {sky }}+(1+A / C) T_{\mathrm{n}}}{\tilde{T}_{\text {ref }}+(1+A / C) T_{\mathrm{n}}}$.

If we relax the hypothesis of completely correlated gain and noise temperature fluctuations then we find:

$$
\begin{aligned}
f_{\mathrm{k}}^{\text {uncorr }}= & \left\{\frac { C } { \tilde { T } _ { \text { sky } } + T _ { \mathrm { n } } } \sqrt { \frac { \beta } { 2 } } \left[\left(\left(\tilde{T}_{\text {sky }}+T_{\mathrm{n}}\right)-r\left(\tilde{T}_{\text {ref }}+T_{\mathrm{n}}\right)\right)^{2}\right.\right. \\
& \left.\left.+(1-r)^{2} T_{\mathrm{n}}^{2}(A / C)^{2}\right]^{1 / 2}\right\},
\end{aligned}
$$

which is minimised (but not nulled) by the following value of $r$ :

$r=r_{\text {uncorr }}^{*}=\frac{\left(\tilde{T}_{\text {sky }}+T_{\mathrm{n}}\right)\left(\tilde{T}_{\text {ref }}+T_{\mathrm{n}}\right)+\left(T_{\mathrm{n}} A / C\right)^{2}}{\left(\tilde{T}_{\text {ref }}+T_{\mathrm{n}}\right)^{2}+\left(T_{\mathrm{n}} A / C\right)^{2}}$.

Note that in both Eqs. (8) and (10) the optimal value of $r$ is dependent on the ratio $C / A \approx 2 \sqrt{N_{\mathrm{s}}}$ where $N_{\mathrm{s}}$ is the number of amplifier stages; if $C / A \gg 1$ then we have that in both cases the optimal value is approximated by $r_{0}^{*}$ (given by Eq. (4)).

In Fig. 3 we show a plot of the knee frequency calculated using Eqs. (7) and (9) versus the relative accuracy $\delta_{r_{0}^{*}}=$ $\left(r-r_{0}^{*}\right) / r_{0}^{*}$ for parameters typical of the $70 \mathrm{GHz}$ radiometer 

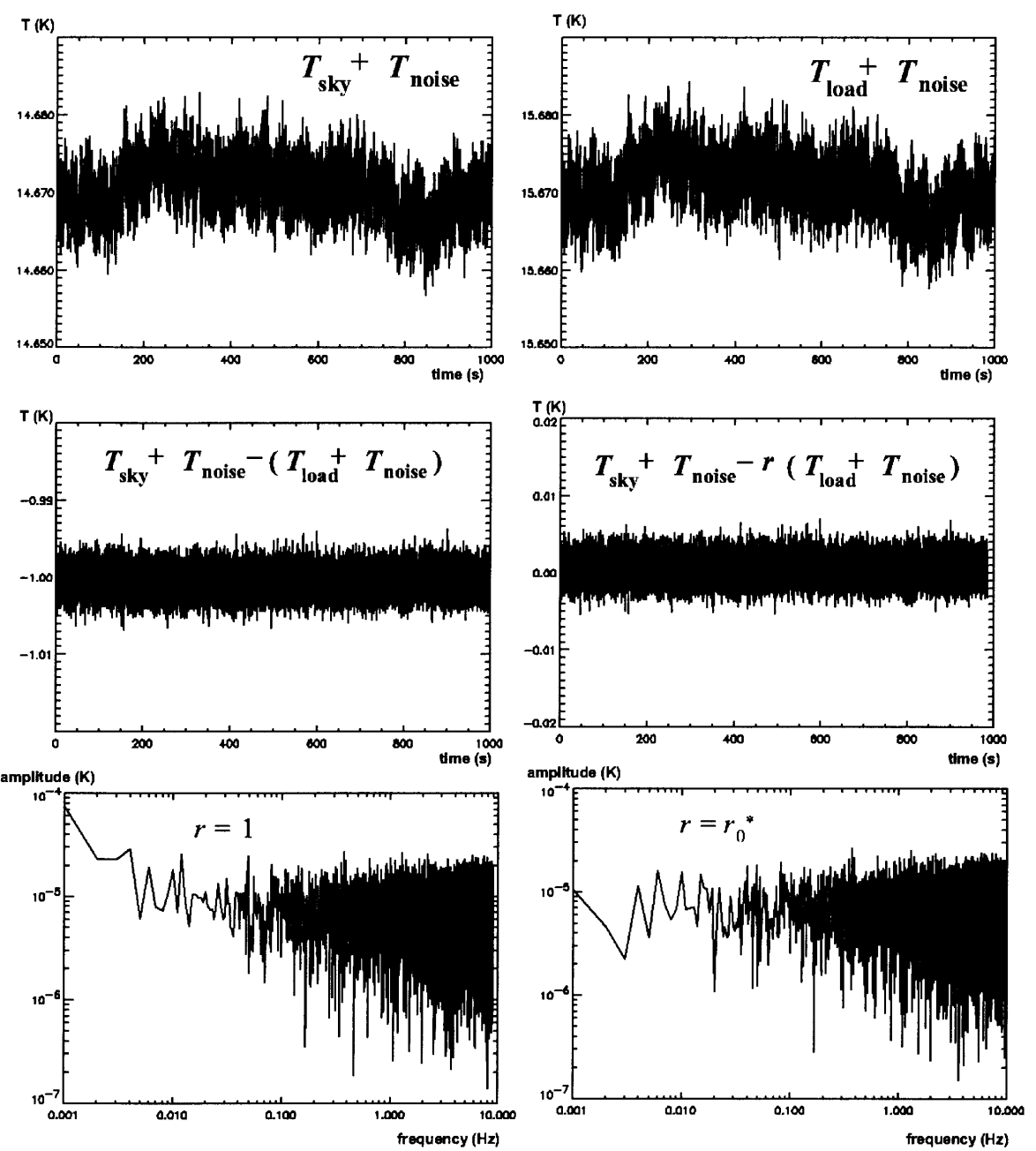

Fig. 4. Simulated noise streams with parameters representative of a a $30 \mathrm{GHz}$ LFI radiometer. The top graphs show the total power data (sky, left panel, and reference load, right panel), the middle graphs the differenced noise with $r=1$ (left panel) and $r=r_{0}^{*}$ and the bottom graphs show the corresponding amplitude spectra.

chain (which drives the accuracy requirements in the calculation of $r$ ).

Two main considerations may be drawn from the results in Fig. 3: first, if $r=r_{0}^{*}$ then the radiometer knee frequency is not nulled also if we assume complete correlation between gain and noise temperature fluctuations. In fact it can be shown (Seiffert et al. 2002) that gain fluctuations are cancelled at first order and the residual $1 / f$ noise comes from noise temperature instabilities. Second, if $r_{0}^{*}$ is known with an accuracy of the order of $\sim 1.5 \%$ then the resulting knee frequency is below the design value $(50 \mathrm{mHz}$ in this case) independently from the hypothesis of correlated or uncorrelated fluctuations in the front-end amplifiers.

In Fig. 4 we show a simulation of the total power and of the differenced noise stream for a $30 \mathrm{GHz}$ LFI radiometer in the hypothesis of correlated gain and noise temperature fluctuations. The simulation has been performed considering typical values for system noise temperature and front-end losses, $\sim 1 \mathrm{~K}$ contribution from the telescope (emissivity of $1 \%$ for each reflector at $50 \mathrm{~K}$ physical temperature), a physical temperature of $4.8 \mathrm{~K}$ for the reference load and a physical temperature of $20 \mathrm{~K}$ for the LFI focal plane.

In the upper graphs of Fig. 4 we show the sky and reference total power data streams, while in the middle graphs we
Table 1. Estimates of $r_{0}^{*}$ for PLANCK-LFI radiometers.

\begin{tabular}{ccc}
\hline \hline $30 \mathrm{GHz}$ & $44 \mathrm{GHz}$ & $70 \mathrm{GHz}$ \\
\hline 0.936 & 0.953 & 0.971 \\
\hline
\end{tabular}

compare the differenced noise streams obtained with $r=1$ (left panel) and $r=r_{0}^{*}$ (right panel); the graphs show that in both cases most of the $1 / f$ instability is removed, although in the $r=1$ case the sky-reference offset is retained. A comparison between the two amplitude spectra (lower panels) reveals that if the ideal gain modulation factor is applied then the final knee frequency is much lower compared to the $r=1$ case $^{2}$.

In Table 1 we provide representative estimates of $r_{0}^{*}$ for all the frequency channels of PLANCK-LFI radiometers.

\subsection{Susceptibility to other systematic effects}

Apart from $1 / f$ noise, other instrumental systematic effects can be mitigated by balancing the offset in the radiometer output. In PLANCK-LFI, for example, systematic effects can be expected

\footnotetext{
2 Note that in the case $r=r_{0}^{*}$ the knee frequency could not be resolved due to data stream length $(1000 \mathrm{~s})$ that limits the frequency resolution to $\sim 5 \mathrm{mHz}$.
} 
from thermal variations of the $20 \mathrm{~K}$ and $300 \mathrm{~K}$ stages and from input bias fluctuations of the front-end amplifiers.

Let us consider, for example, the effect caused by thermal instabilities in the front-end and in the back-end stages of the PLANCK-LFI radiometers. Variations in the physical temperature in the radiometer couple with the measured output essentially through the following mechanisms:

- variations in thermal noise induced by resistive front-end passive components (feed-horn, OMT and first hybrid);

- amplifier gain oscillations (in the front-end and in the back-end);

- amplifier noise temperature oscillations (in the front-end ${ }^{3}$ ).

The radiometer susceptibility can be written in terms of a transfer function, $\phi$, that links a physical temperature variation, $\delta T_{\text {phys }}$, to the corresponding variation in the measured output, $\delta T_{\text {out }}$, i.e.: $\delta T_{\text {out }}=\phi \times \delta T_{\text {phys }}$. This function has been calculated following the approach discussed in Seiffert et al. (2002) assuming complete correlation between the temperature fluctuations at the level of the various radiometer components. This assumption is a reasonable one in the context of the PLANCK-LFI radiometers where physical temperature variations induced in the $20 \mathrm{~K}$ and $300 \mathrm{~K}$ stages will be dominated by relatively "slow" components with a very small contribution from harmonics with a frequency higher than $\sim 5 \mathrm{mHz}$.

Under these assumptions the susceptibility to temperature variations in the $20 \mathrm{~K}$ stage $\left(\phi_{\mathrm{FE}}\right)$ and in the $300 \mathrm{~K}$ back-end stage $\left(\phi_{\mathrm{BE}}\right)$ can be written as:

$$
\begin{aligned}
\phi_{\mathrm{FE}}= & L_{\mathrm{sky}}\left\{\left(1-L_{\mathrm{sky}}^{-1}\right)-r\left(1-L_{\mathrm{ref}}^{-1}\right)\right. \\
& +\frac{\ln (10)}{10}\left[\tilde{T}_{\mathrm{sky}}+T_{\mathrm{n} F \mathrm{~F}}-r\left(\tilde{T}_{\mathrm{ref}}+T_{\mathrm{nFE}}\right)\right] \frac{\partial G_{\mathrm{FE}}^{\mathrm{dB}}}{\partial T_{\mathrm{phys}}} \\
& \left.+(1-r) \frac{\partial T_{\mathrm{n}_{\mathrm{FE}}}}{T_{\mathrm{phys}}}\right\}, \\
\phi_{\mathrm{BE}}= & L_{\mathrm{sky}} \frac{\ln (10)}{10}\left(\frac{\partial a^{\mathrm{dB}}}{\partial T_{\mathrm{phys}}}+\frac{\partial G_{\mathrm{BE}}^{\mathrm{dB}}}{\partial T_{\mathrm{phys}}}\right) \\
& \times\left[\tilde{T}_{\mathrm{sky}}+T_{\mathrm{sys}}-r\left(\tilde{T}_{\mathrm{ref}}+T_{\mathrm{sys}}\right)\right],
\end{aligned}
$$

where $G_{\mathrm{FE}}^{\mathrm{dB}}$, and $T_{\mathrm{n}}$ are the gain in $\mathrm{dB}$ and the noise temperature of the front-end amplifiers, $G_{\mathrm{BE}}^{\mathrm{dB}}$ the gain of the backend amplifiers, $a^{\mathrm{dB}}$ the diode constant and $T_{\text {phys }}$ the physical temperature.

Note from Eq. (12) that the susceptibility to back end temperature fluctuations is cancelled if the radiometer output is balanced (i.e. $\phi_{f_{\mathrm{BE}}}=0$ for $r=r_{0}^{*}$ ); this is not only true for temperature variations, but for also for any instrumental effect causing instability in the back-end amplifiers.

The susceptibility to front-end temperature variations, instead, is cancelled for a value of $r$ which is slightly different from $r_{0}^{*}$ (its expression is not reported here, but it can be derived from $\phi_{\mathrm{FE}}=0$ in Eq. (11)). In general the effect of any

\footnotetext{
3 The $\sim 35 \mathrm{~dB}$ gain in the front-end makes negligible the effect from fluctuations in the noise temperature of the back-end amplifiers.
}

given systematic effect in the radiometer can be cancelled at first order using a particular value of the gain modulation factor; in practice, however, the choice of the "best" value of $r$ to use in the difference is driven by the most critical expected effect and by the feasibility to calculate $r$ from the measured data with the required accuracy.

In PLANCK-LFI, for example, with the condition $r=r_{0}^{*}$ it is possible to minimise $1 / f$ amplifier noise and effects from the back-end while keeping the radiometric susceptibility to other systematic effects at a level which allows to define realistic requirements on the thermal and electrical stability at the interfaces between the satellite and the instrument.

\subsection{Required accuracy in the calculation of $r_{0}^{*}$}

The main goal for balancing the radiometer output is to minimise the residual $1 / f$ noise; therefore it is natural that the requirement on the gain modulation factor accuracy must be derived from the maximum knee frequency allowed in the experiment.

From the curves shown in Fig. 3 we have derived a requirement on the accuracy of the gain modulation factor of the order of $\sim \pm 1.5 \%$.

In some cases, however, the accuracy needed in the calculation of the gain modulation factor may be determined by the level of other effects; in PLANCK-LFI radiometers, for example, the value $r_{0}^{*}$ will not only minimise $1 / f$ noise, but also effects from back-end thermal instabilities; in this case the need for a very low radiometer susceptibility to back-end temperature fluctuations imposes a tight requirement on the accuracy of the gain modulation factor, ranging from $\sim \pm 1 \%$ at $30 \mathrm{GHz}$ to $\sim \pm 0.3 \%$ at $70 \mathrm{GHz}$.

\section{Calculation of $r_{0}^{*}$ from measured data}

During a long-duration CMB measurement the radiometer noise properties are subject to slight, slow variations in time due to thermal/electrical drifts and ageing of the radiometer electronic components. If the offset balancing is done in software, then the value of $r_{0}^{*}$ can be recalculated and updated (if necessary) in the data reduction software to compensate for such changes.

As we show in detail in this section, the gain modulation factor can be calculated from radiometer data acquired in "total power mode", i.e. before differencing is performed. Although the current telemetry bandwidth available to the LFI instrument allows to download the complete total power data streams, it is possible to calculate $r$ from a limited portion (about $\sim 15 \mathrm{~min}$ ) of these data. This implies that it could be possible to perform the sky-load differencing on-board (with a saving of $\sim 50 \%$ in telemetry bandwidth) providing margin for possible nonnominal operation scenarios.

In this section we discuss the following three calculation methods and compare them from the point of view of the obtainable accuracy:

1. $r_{0}^{*}$ calculated from the ratio of the average sky and reference load levels; 
2. $r_{0}^{*}$ calculated from the ratio of the sky and reference load standard deviations;

3. $r_{0}^{*}$ calculated by minimising of the final differenced data stream knee frequency.

In the following discussion we will consider only the presence of white $+1 / f$ noise in the radiometer data streams and assume the availability of $15 \mathrm{~min}$ of total power data (as in the case of PLANCK-LFI) in order to evaluate the efficiency of each method. The effect of the presence of the astrophysical signal and of other instrumental systematic effects is discussed in Sect. 6.

\subsection{Calculation from average signal level}

The simplest way to calculate $r$ from the total power data is to use directly the definition provided by Eq. (4), i.e. to take the ratio between the average signals acquired when looking at the sky, $V_{\text {sky }, i}=G_{i}\left(\tilde{T}_{\text {sky }}+T_{\mathrm{n}, i}\right)$, and when looking at the reference load, $V_{\mathrm{ref}, i}=G_{i}\left(\tilde{T}_{\mathrm{ref}}+T_{\mathrm{n}, i}\right)$. In this case $r$ is given by:

$r=\frac{\sum_{i=1}^{N} V_{\mathrm{sky}, i}}{\sum_{i=1}^{N} V_{\mathrm{ref}, i}}$

where $N$ represents the number of samples available in the data stream. Now we know that although gain and the noise temperature will display $1 / f$ fluctuations, thanks to the pseudocorrelation scheme they will be the same (at first order) in both the sky and reference signals. Therefore for each sample, $i$, we can write: $G_{i}=G+\delta G_{i}^{1 / f}$ and $T_{\mathrm{n}, i}=T_{\mathrm{n}}+\delta T_{\mathrm{n}, i}^{1 / f}$, where $G$ and $T_{\mathrm{n}}$ represent the average gain and noise temperature level. Furthermore there will be a white nose component $\delta T_{\text {sky(ref }}^{\mathrm{WN}}$ such that $\left\langle\delta T_{\text {sky(ref) }}^{\mathrm{WN}}\right\rangle=0$ and $\sigma_{\text {sky(ref) }}^{\mathrm{WN}}=\left(\tilde{T}_{\text {sky(ref })}+T_{\mathrm{n}}\right) / \sqrt{\beta \tau}$.

Let us now evaluate the impact of gain and noise temperature fluctuations separately. If the total power noise streams contain $1 / f$ gain fluctuations then we can write the sky and load data streams as:

$V_{\mathrm{sky}(\mathrm{ref}), i}=\left(G+\delta G_{i}^{1 / f}\right)\left(\tilde{T}_{\mathrm{sky}(\mathrm{ref})}+T_{\mathrm{n}}\right)+\delta T_{\mathrm{sky}(\mathrm{ref})}^{\mathrm{WN}} ;$

therefore the sums in Eq. (13) have the form:

$$
\begin{aligned}
\sum_{i=1}^{N} V_{\text {sky(ref }), i}= & G N\left(\tilde{T}_{\text {sky(ref })}+T_{\mathrm{n}}\right) \\
& \times\left[1+\frac{1}{N} \sum_{i=1}^{N} \frac{\delta G_{i}^{1 / f}}{G}\right]+\sum_{i=1}^{N} \delta T_{\text {sky(ref) }}^{\mathrm{WN}}
\end{aligned}
$$

Similarly, in the case of $1 / f$ noise temperature fluctuations then Eq. (15) becomes:

$$
\begin{aligned}
\sum_{i=1}^{N} V_{\text {sky(ref }), i}= & G N\left(\tilde{T}_{\text {sky(ref })}+T_{\mathrm{n}}\right) \\
& \times\left[1+N^{-1} \sum_{i=1}^{N} \frac{\delta T_{\mathrm{n}, i}^{1 / f}}{\tilde{T}_{\text {sky(ref })}+T_{\mathrm{n}}}\right] \\
& +\sum_{i=1}^{N} \delta T_{\text {sky(ref })}^{\mathrm{WN}} .
\end{aligned}
$$

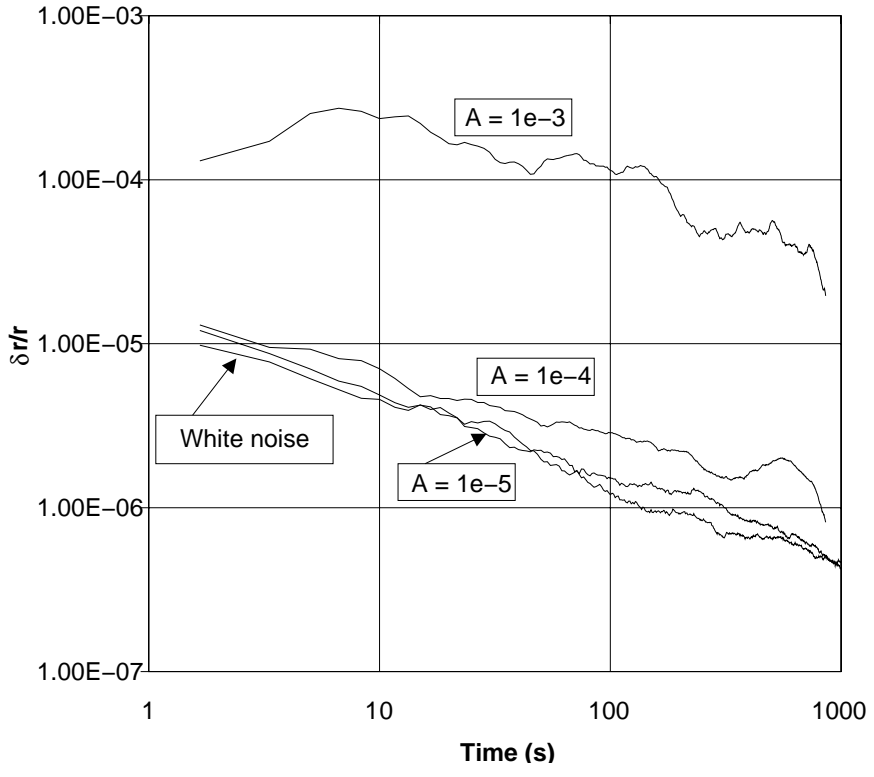

Fig. 5. Effect of gain and noise temperature $1 / f$ fluctuations on the calculation of $r$ from the ratio of the average signal levels. Each curve is an average of 50 noise realisations relative to the $30 \mathrm{GHz}$ PLANCK-LFI channel.

In both Eqs. (15) and (16) the two sums on the right-hand side tend to zero for large values of $N$, so that the ratio of the average signals $\rightarrow r_{0}^{*}$ for $N \rightarrow \infty$, which implies that Eq. (13) can be used also in presence of $1 / f$ noise provided that the statistics is sufficiently large.

In Fig. 5 we show the result of a numerical simulation of the convergence of Eq. (13) to the theoretical value $r_{0}^{*}$. Each curve represents the value of $\delta r / r_{0}^{*}$ versus time, averaged over 50 equivalent noise realisations of the $30 \mathrm{GHz}$ PLANCK-LFI channel. The different curves are relative to different levels of $1 / f$ noise in both gain and noise temperature ( $A=0$, i.e. white noise only, $A=10^{-5}, A=10^{-4}, A=10^{-3}$ i.e. low, intermediate and high level of $1 / f$ noise). The figure shows that the presence of $1 / f$ fluctuations determines a slower convergence with respect to the white noise case. Even in the worst case, however, (which represents an unrealistic case with respect to the expected LFI performances) the accuracy obtained with 15 min of data is much better than $0.01 \%$.

In order to calculate properly the ratio $r$ from Eq. (13), it is necessary that any "zero-level error" introduced by the backend electronics is small. Let us evaluate the effect of a constant offset $\delta V$ added to both states in the switching output of the radiometer; the voltage measured in the two switch states can be written as:

$V_{\text {sky(ref) }}=G\left(\tilde{T}_{\text {sky(ref })}+T_{\mathrm{n}}\right)+\delta V$.

If the offset is unknown, it introduces an uncertainty given by:

$\delta r=\frac{V_{\text {sky }}}{V_{\text {ref }}}-\frac{V_{\text {sky }}+\delta V}{V_{\text {ref }}+\delta V}=\frac{V_{\text {sky }}-V_{\text {ref }}}{V_{\text {ref }}\left(V_{\text {ref }}+\delta V\right)} \delta V$.

From Eq. (18) it is straightforward to obtain the maximum values of the unknown offsets that would produce an uncertainty on $r$ equal to the required accuracy. In the case of PLANCK-LFI these values (in temperature units) range 
from $\sim \pm 0.5 \mathrm{~K}$ at $30 \mathrm{GHz}$ to $\sim \pm 1.36 \mathrm{~K}$ at $70 \mathrm{GHz}$, which are compatible with the current electronics design.

Finally we underline that the above treatment is valid in the hypothesis of detector linearity and negligible gain compression. In general, however, these conditions are largely met in $\mathrm{CMB}$ anisotropy experiments, where the signals to be measured are small. In the case of the PLANCK-LFI radiometers, for example, the radiometer output power is of the order of $-25 \mathrm{dBm}$, which is much smaller than the $-1-\mathrm{dB}$ compression point of the HEMT amplifiers that is of the order of $\sim-15 \mathrm{dBm}$ and falls well in between the range of detector linearity $(\sim-35 \div-15 \mathrm{dBm})$.

\subsection{Calculation from standard deviations}

A second approach is to calculate $r$ from the ratio of the standard deviations of the total power noise streams, i.e.:

$$
r=\frac{\sigma_{\mathrm{sky}}^{N}}{\sigma_{\mathrm{ref}}^{N}}=\left[\frac{\sum_{i=1}^{N}\left(V_{\mathrm{sky}, i}-\left\langle V_{\mathrm{sky}}\right\rangle\right)^{2}}{\sum_{i=1}^{N}\left(V_{\mathrm{ref}, i}-\left\langle V_{\mathrm{ref}}\right\rangle\right)^{2}}\right]^{1 / 2}
$$

An advantage of this method compared to the previous one is that the ratio of standard deviations is insensitive to spurious offsets introduced by the back-end electronics; let us evaluate now the accuracy that is obtainable in presence of noise. In the case the data streams are characterised by white noise only then we have that $\sigma_{\text {sky(ref) }}^{N} \rightarrow\left(\tilde{T}_{\text {sky(ref })}+T_{\mathrm{n}}\right) / \sqrt{\beta \tau}$ for $N \rightarrow \infty$, so that for large values of $N$ Eq. (19) approximates $r_{0}^{*}$.

Of couse, it is also well known that the standard deviation of an infinite time stream of $1 / f$-type noise formally diverges. For the case under consideration here, the ratio of standard deviations of finite sets of white noise plus some $1 / f$-type noise, we examine below the details of accuracy and convergence.

Let us consider first the effect of gain fluctuations; from Eq. (15) we have that

$$
\begin{aligned}
\left\langle V_{\text {sky(ref })}\right\rangle_{N}= & \frac{1}{N} \sum_{i=1}^{N} V_{\text {sky(ref) }, i} \\
= & G\left(\tilde{T}_{\text {sky(ref) }}+T_{\mathrm{n}}\right)\left(1+\left\langle\frac{\delta G^{1 / f}}{G}\right\rangle_{N}\right) \\
& +\left\langle\delta T_{\text {sky(ref) }}^{\mathrm{WN}}\right\rangle_{N},
\end{aligned}
$$

and, consequently,

$$
\begin{aligned}
& \sum_{i=1}^{N}\left(V_{\text {sky(ref }), i}-\left\langle V_{\text {sky(ref })}\right\rangle\right)^{2} \approx \\
& \left(\tilde{T}_{\text {sky(ref })}+T_{\mathrm{n}}\right)^{2} \sum_{i=1}^{N}\left(\delta G_{i}^{1 / f}-\left\langle\delta G^{1 / f}\right\rangle\right)^{2} \\
& +\sum_{i=1}^{N}\left(\delta T_{\text {sky(ref }), i}^{\mathrm{WN}}-\left\langle\delta T_{\mathrm{sky}(\mathrm{ref})}^{\mathrm{WN}}\right\rangle\right)^{2} \\
& \stackrel{N \rightarrow \infty}{\longrightarrow}(N-1)\left[\left(\tilde{T}_{\mathrm{sky}(\mathrm{ref})}+T_{\mathrm{n}}\right)^{2} \sigma_{G^{1 / f}}^{2}+\left(\sigma_{\mathrm{sky}(\mathrm{ref})}^{\mathrm{WN}}\right)^{2}\right]
\end{aligned}
$$

Considering that $\sigma_{\text {sky(ref })}^{\mathrm{WN}} \stackrel{N \rightarrow \infty}{\longrightarrow}\left(\tilde{T}_{\text {sky(ref })}+T_{\mathrm{n}}\right) / \sqrt{\beta \tau}$ then it follows that

$\sigma_{\text {sky(ref) }}^{N} \stackrel{N \rightarrow \infty}{\longrightarrow}\left(\tilde{T}_{\text {sky(ref })}+T_{\mathrm{n}}\right)\left(\sigma_{G^{1 / f}}+\frac{1}{\sqrt{\beta \tau}}\right)$,

so that the ratio of the total power standard deviations converges to $r_{0}^{*}$ for large $N$.

Let us now consider the presence of $1 / f$ fluctuations in the noise temperature. In this case Eq. (21) has the form:

$$
\begin{aligned}
& \sum_{i=1}^{N}\left(V_{\text {sky(ref) }, i}-\left\langle V_{\text {sky(ref })}\right\rangle\right)^{2} \approx G^{2} \sum_{i=1}^{N}\left(\delta T_{\mathrm{n}, i}^{1 / f}-\left\langle\delta T_{\mathrm{n}}^{1 / f}\right\rangle\right)^{2} \\
& +\sum_{i=1}^{N}\left(\delta T_{\text {sky(ref) }, i}^{\mathrm{WN}}-\left\langle\delta T_{\mathrm{sky}(\mathrm{ref})}^{\mathrm{WN}}\right\rangle\right)^{2} \\
& \stackrel{N \rightarrow \infty}{\longrightarrow}(N-1)\left[G^{2} \sigma_{T_{\mathrm{n}}^{1 / f}}^{2}+\left(\sigma_{\mathrm{sky}(\mathrm{ref})}^{\mathrm{WN}}\right)^{2}\right] .
\end{aligned}
$$

It follows that the ratio of standard deviations converges, in this case, to:

$$
\sqrt{\frac{G^{2} \sigma_{T_{\mathrm{n}}^{1 / f}}^{2}+\left(\sigma_{\mathrm{sky}}^{\mathrm{WN}}\right)^{2}}{G^{2} \sigma_{T_{\mathrm{n}}^{1 / f}}^{2}+\left(\sigma_{\mathrm{ref}}^{\mathrm{WN}}\right)^{2}}}=r_{0}^{*} \sqrt{\frac{\left(G \sigma_{T_{\mathrm{n}}^{1 / f}} / \sigma_{\mathrm{sky}}^{\mathrm{WN}}\right)^{2}+1}{\left(G \sigma_{T_{\mathrm{n}}^{1 / f}} / \sigma_{\mathrm{ref}}^{\mathrm{WN}}\right)^{2}+1}} \neq r_{0}^{*} .
$$

This clearly shows that in presence of noise temperature $1 / f$ fluctuations the ratio of standard deviations does not converge to $r_{0}^{*}$. In particular with increasing amplitude of the $1 / f$ noise temperature fluctuations (i.e. for increasing values of $A$ ) we have that $G \sigma_{T_{\mathrm{n}}}^{1 / f} \gg \sigma_{\text {sky(ref) }}^{\mathrm{WN}}$ and the ratio of standard deviations tends to 1 . We can therefore calculate the following lower limit for the relative accuracy on $r$ obtained by this method:

$\frac{\delta r}{r} \stackrel{A \rightarrow \infty}{\longrightarrow} \frac{1-r_{0}^{*}}{r_{0}^{*}}$

In Fig. 6 we show the results of numerical simulations relative to the $30 \mathrm{GHz}$ PLANCK-LFI channel showing the convergence of the ratio of noise standard deviations (Eq. (19)) for various levels of $1 / f$ noise. The two dashed curves represent the limits for $A \rightarrow 0$ (i.e. white noise only) and $A \rightarrow \infty$. In the first case (white noise), because the standard deviation of $N$ random samples tends to the standard deviation of the gaussian distribution as $\delta \sigma / \sigma=1 / \sqrt{N}$, we have that the accuracy calculated by the ratio of the standard deviations increases with the number of samples as $\delta r / r=2 / \sqrt{N}$. In the second case we have that the ratio of standard deviations does not converge to $r_{0}^{*}$ but to $\frac{1-r_{0}^{*}}{r_{0}^{*}}$. The figure clearly shows that for increasing values of $A$ the behaviour changes between these two limits.

When the $1 / f$ noise level is high it is still possible to estimate $r$ from the ratio of the white noise rms that can be derived either by fitting the high frequency end of the noise power spectrum or using a proxy of the white noise level by means of the two-point variance function $\sigma^{2}(i-j)=\left\langle\left(x_{i}-x_{j}\right)^{2}\right\rangle$ as described by Janssen et al. (1996). In this case $r$ will converge to $r_{0}^{*}$ with an upper limit accuracy of $\sim 2 / \sqrt{N}$ (see Fig. 6). 


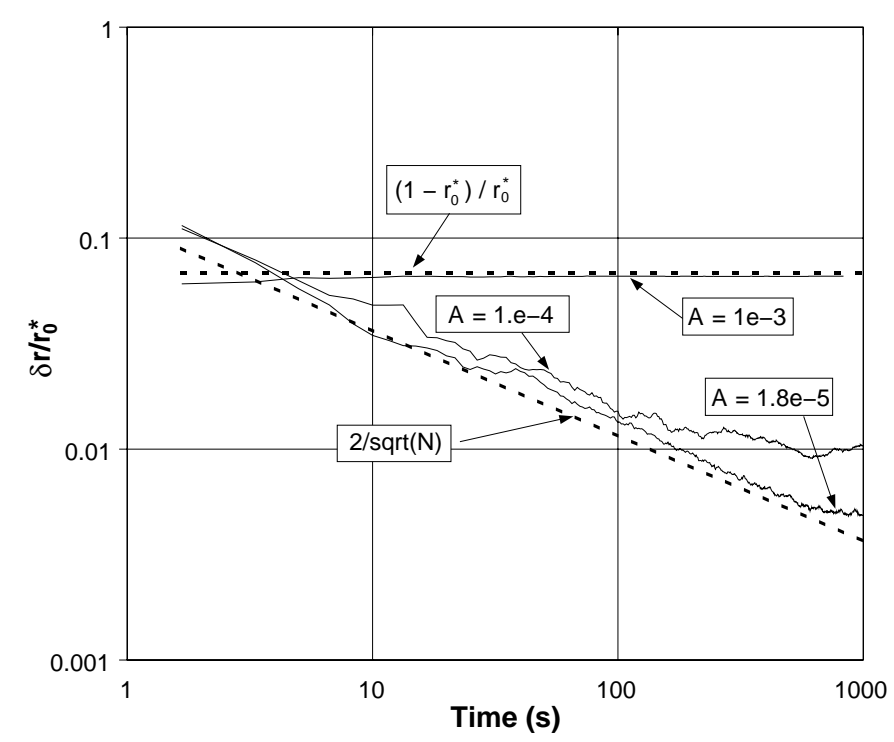

Fig. 6. Effect of noise temperature $1 / f$ fluctuations on the calculation of $r$ from the ratio of standard deviations signal levels. Each curve is an average of 50 noise realisations relative to the $30 \mathrm{GHz}$ PLANCK-LFI channel.

\subsection{Calculation by minimising the final knee frequency}

A third approach that we consider here is the optimisation of the gain modulation factor by defining a window $\left[r_{\min }, r_{\max }\right]$ around $r_{0}^{*}$ and then finding the "best" value of $r$ that minimises knee frequency of the differenced noise stream.

Although this method probes directly the $1 / f$ noise characteristics of the final differenced data, there are some limitations and caveats that are worth mentioning:

1. Frequency resolution and accuracy. If $\Delta t$ represents the length (in time) of the data available for analysis, then the absolute minimum frequency that can be resolved in Fourier space is $f_{\min } \sim 2 / \Delta t$. For $\Delta t \sim 900 \mathrm{~s}(15 \mathrm{~min})$ we have that $f_{\min } \sim 2 \mathrm{mHz}$. Although the data length can be enough to resolve frequencies $\sim 1 \mathrm{mHz}$, in order to determine $f_{\mathrm{k}}$ we must be able to recognise the frequency at which the $1 / f$ noise has an amplitude equal to the rms value of the white noise. Because noise dominates, each individual sample has $100 \%$ uncertainty so that we must rebin the data with a consequent loss in frequency resolution.

2. Presence of astrophysical signal in differenced noise stream. When the sky and load data streams are differenced taking into account a value of $r$ in the interval $\left[r_{\min }, r_{\max }\right]$, the differenced noise stream will also contain the astrophysical signal (dipole, galaxy, etc.) at all the harmonics of the scan frequency $(\sim 16 \mathrm{mHz}$ for PLANCK). This implies that with the real data the calculation of the knee frequency will be affected, in principle, by the power at the first 10 harmonics or so of the scan frequency. Therefore some assumptions on the expected signal are probably necessary to remove it from the differenced data before computing $f_{\mathrm{k}}$.

\section{Application to laboratory radiometer data}

The gain modulation strategy described in the previous sections is routinely applied with success in the PLANCK-LFI prototype radiometers. Here we present some results obtained with experimental data from the $30 \mathrm{GHz}$ LFI Elegant BreadBoard (EBB) radiometer assembled at the Jodrell Bank Observatory (JBO) in the framework of the PLANCK collaboration. Further details about the EBB testing campaign of the PLANCK-LFI radiometers will be discussed in a dedicated set of papers which is currently in preparation.

The EBB $30 \mathrm{GHz}$ radiometer is comprised of a frontend module (FEM) manufactured at JBO and a back-end module (BEM) manufactured at the University of Cantabria (Santander, Spain) connected by waveguides approximately $0.75 \mathrm{~m}$ long. Each FEM channel includes a HEMT phase switch which has also been designed and manufactured by JBO. In the tests described here the FEM was operated at $20 \mathrm{~K}$ while the BEM was maintained at room temperature.

Because the aim of these tests was to verify the validity of the differential concept rather than to achieve flight-like performances, the test setup was kept as simple as possible; in particular the front-end phase switches were operated at $280 \mathrm{~Hz}$ (instead of $4096 \mathrm{~Hz}$ as in the LFI baseline) and the $300 \mathrm{~K}$ thermal environment was not maintained at a high degree of stability.

Before the stability tests were carried out, the BEM white noise floor was measured to be significantly less than the noise level with the FEM and BEM connected together. Fifteen minute tests were then carried out with one of the phase switches kept in a fixed state. A $280 \mathrm{~Hz}$ square wave switch waveform for the other phase switch was generated by the data acquisition card counter outputs, under LabVIEW control. The detected signals were therefore $280 \mathrm{~Hz}$ square waves, which were sampled at 504000 samples per second. After discarding $20 \%$ of the data ( $10 \%$ from each edge of the square wave), each switched state was averaged to obtain a single number, $A_{1}, B_{1}, A_{2}$ or $B_{2}$, where A represents the "on" state for each channel and $B$ the "off" state.

In the left graph of Fig. 7 we show the total power levels of one of the two output radiometer detectors, the top trace representing the $A$ states and the lower the $B$ states. The small inset shows how the actual averaged diode output looks, i.e. a series of $A$ and $B$ values alternating at half the frequency of the phase switch; in the big graph, instead, the measured values relative to the two switch states are shown separately for better clarity.

From the ratio of the average levels of the two data streams we have calculated a value $r=0.96075$ that has been applied to produce the differenced noise stream shown in the top panel of the right part of Fig. 7. The graph shows that the offset has been removed as well as the long-timescale instabilities that are evident in the total power data. The power spectrum of the differenced data shows a final knee frequency of the order of $\sim 40 \mathrm{mHz} .^{4}$

We also calculated $r$ by taking the ratio of the standard deviation of the data streams, and by directly minimising the

\footnotetext{
4 The knee frequency was defined as the frequency at which the power spectrum has a value equal to $\sqrt{2}$ of the average white noise level.
} 


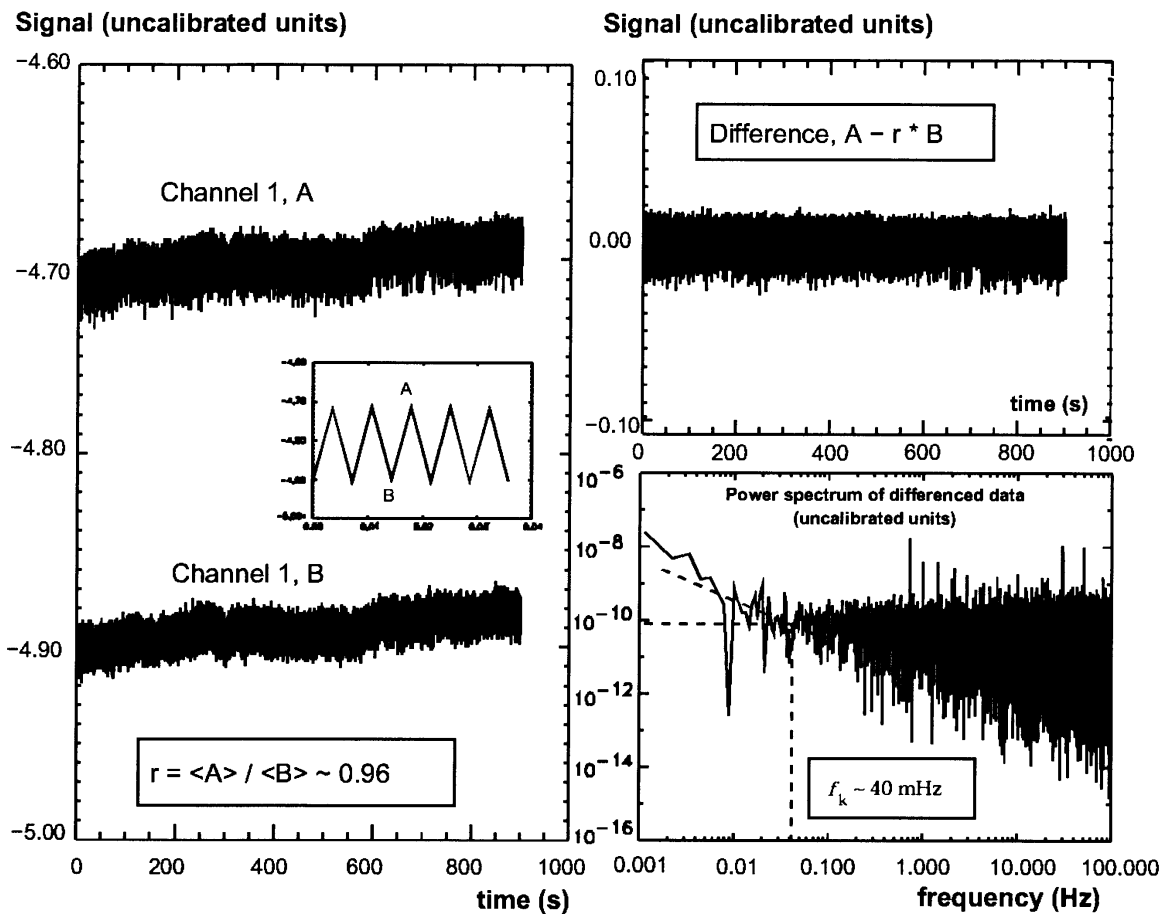

Fig. 7. Measured data from the $30 \mathrm{GHz}$ PLANCK-LFI prototype radiometer. The graph on the left shows about 15 min of total power noise data from one of the two radiometer channels. The small inset shows how measurements actually appear at the output of each detector, i.e. as an alternating series of $A$ and $B$ values. The two panels on the right show the differenced noise stream in time and frequency domain. The final knee frequency is $\sim 40 \mathrm{mHz}$.

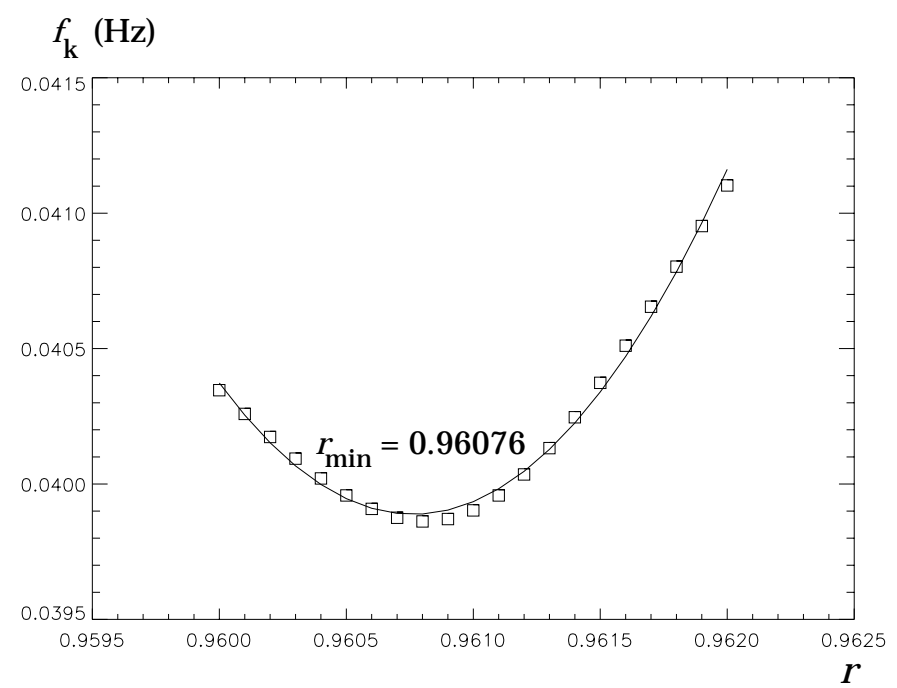

Fig. 8. Behaviour of $f_{\mathrm{k}}$ versus $r$ around the minimum. The curve was obtained by applying different values of $r$ to the total power data shown in Fig. 7 and then calculating the knee frequency of the differenced data. The continuous line is a polynomial fit of the discrete data points.

final knee frequency. In the first case we obtained a value of $r=0.95568$, while in the second case we found $r=0.96076$ which is practically coincident with the one calculated with the ratio of signal levels.

In Fig. 8 we show the behaviour of the knee frequency versus $r$ around the minimum; the continuous line represents a polynomial fit used to determine the minimum.

Although the knee frequency obtained from the differential data is within the PLANCK-LFI requirements its value is higher than the theoretical value $(\lesssim 10 \mathrm{mHz})$ that can be estimated from Eqs. (7) and (9).
Part of this discrepancy was caused by thermal drifts in the $300 \mathrm{~K}$ environment (that were observed to be of the order of $1.3 \mathrm{~K} / \mathrm{hr}$ and can be clearly seen in the total power data streams); to evaluate this effect we removed the linear trend from the data and then calculated the spectrum of the resulting differenced time stream. In Fig. 9 we show the total power and the differential radiometric outputs after the removal of the linear trends; the noise power spectrum shows that the final knee frequency was reduced from $\sim 40$ to $\sim 26 \mathrm{mHz}$.

The remaining discrepancy of about $\sim 15 \mathrm{mHz}$ was likely to be caused by other non-idealities in the experimental setup which have not been completely understood yet.

These results demonstrate how the pseudo-correlation scheme adopted for the LFI radiometers is effective in reducing the effect of amplifier instability to very low levels also in experimental conditions far from the nominal flight operations. Furthermore, we have shown that the gain modulation factor can be determined with very simple analysis of a limited portion of the total power radiometer data.

\section{Impact of main systematic effects on the calculation of $r$}

In this section we present a brief analysis of the impact of some systematic effects on the calculation of the gain modulation factor. We can identify the following three possible classes of effects that can impact on the calculation of $r$ :

1. Effects that induce a variation of the total power signal levels, which have an influence especially in the case $r$ is calculated using the ratio of the average total power sky and load levels; 


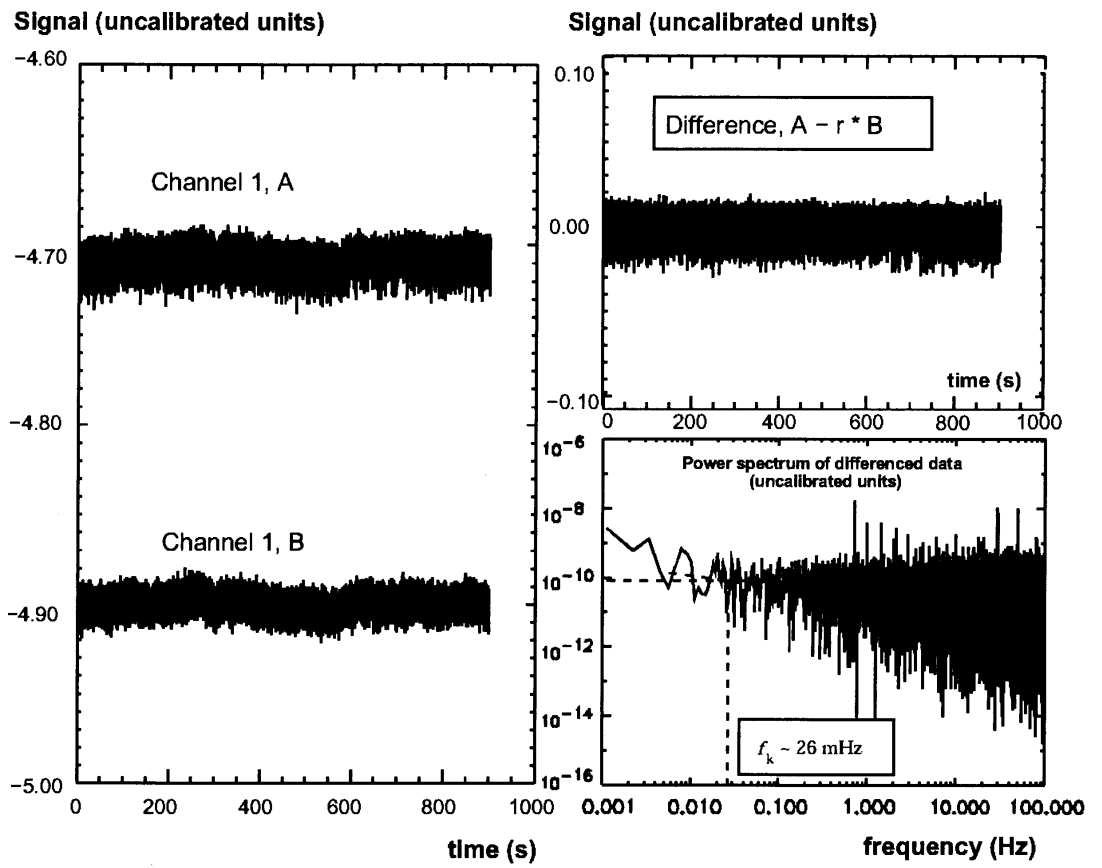

Fig. 9. Left panel: total power radiometer data after the removal of the linear trend. Right panel: differetial output in time (top) and frequency domains (power spectrum, bottom). The knee frequency was estimated $\sim 26 \mathrm{mHz}$.
2. Effects that induce a variation of the standard deviation of the total power noise streams, which have an effect in the case $r$ is calculated using the ratio of standard deviations;

3. Effects that change the low frequency spectral behaviour of the noise streams and limit the ability to derive $r$ from the analysis of the differenced noise stream frequency spectrum.

Considering that the most accurate method to derive $r$ is from the ratio of the average signal levels, we focus on the first class of effects, which can be subdivided into the two following sub-classes:

1. Effects that induce roughly the same variation in both the sky and reference load total power signals (symmetric effects), and

2. Effects that introduce variations in only one of the two signals (sky or reference load, asymmetric effects).

Effects that belong to the first category are, for example, fluctuations induced by radiometer temperature instabilities and by bias voltage variations. Effects belonging to the second category are, for example, the CMB dipole, telescope temperature fluctuations, reference load variations.

\subsection{Symmetric effects}

Let us consider a systematic effect that causes a variation $\delta T$ in both the sky and reference load total power signals. Clearly this is completely equivalent to the case of a constant offset introduced by the back-end electronics discussed in Sect. 4.1. Therefore we can conclude that we need systematic effects with an amplitude of the order of $1 \mathrm{~K}$ (which is 2-3 order of magnitudes higher compared to what is expected for PLANCK-LFI) or more to to introduce uncertainties in the value of $r$ greater than $1 \%$.

\subsection{Asymmetric effects}

Let us now consider a systematic effect that causes a variation $(\delta T)^{\text {sky }}$ only in the sky power signal. The relative uncertainty on the value of $r$ determined by this effect is:

$$
\frac{\delta r}{r_{0}^{*}}=\frac{(\delta T)^{\mathrm{sky}}}{\tilde{T}_{\mathrm{sky}}+T_{\mathrm{n}}} .
$$

In the case we have a systematic variation only in the reference load signal then the uncertainty on $r$ is:

$$
\frac{\delta r}{r_{0}^{*}}=\frac{(\delta T)^{\mathrm{ref}}}{\tilde{T}_{\mathrm{ref}}+T_{\mathrm{n}}+(\delta T)^{\mathrm{ref}}} .
$$

In Table 2 we report, for the PLANCK-LFI 30 and $100 \mathrm{GHz}$ channels, the values of the sky and reference load variations that would determine a change in $r$ equal to the needed accuracy (first two rows), together with estimates of the variation in the sky and reference load signals due to the major expected asymmetric effects (last three rows).

The values reported in Table 2 show that in the PLANCK-LFI case the calculation of the gain modulation factor from the ratio of the average signal level is largely immune from any expected systematic variations in the sky signal and/or in the reference load. This means that in this case $r$ can be considered constant at first order and the only significant changes are expected from long timescale variations of the radiometer noise properties. These changes will be recognised in the ground data analysis and compensated for by periodically updating the value of $r$ during data reduction.

\section{Conclusions}

High sensitivity $\mathrm{CMB}$ radiometric measurements require a very low susceptibility of the receiver to amplifier $1 / f$ noise, which can be obtained only by differential measurements. The 
Table 2. Impact of asymmetric effects on the calculation of the gain modulation factor for the PLANCK-LFI radiometers. The first two rows show the variation in the sky and reference signal that would cause a change in $r$ equal to the needed accuracy of $\pm 1 \%$ at $30 \mathrm{GHz}$ and of $\pm 0.3 \%$ at $70 \mathrm{GHz}$. The last three rows list the expected sky and reference signal variations caused by the major sources of asymmetric effects (CMB dipole, telescope temperature fluctuations, reference load instabilities).

\begin{tabular}{ccc}
\hline \hline & $30 \mathrm{GHz}$ & $70 \mathrm{GHz}$ \\
\cline { 2 - 3 } & \multicolumn{2}{c}{ values in $\mathrm{mK}$} \\
\hline$(\delta T)_{\max }^{\text {sky }}$ & \pm 146 & \pm 161 \\
\hline$(\delta T)_{\max }^{\text {ref }}$ & \pm 154 & \pm 165 \\
\hline \hline$(\delta T)_{\text {dip. }}$ & \pm 3.4 & \pm 3.1 \\
\hline$(\delta T)_{\text {tel. }}$ & $< \pm 1$ & $< \pm 1$ \\
\hline$(\delta T)_{\text {ref }}$ & $< \pm 1$ & $< \pm 1$ \\
\hline
\end{tabular}

pseudo-correlation differential radiometer is a receiver particularly suitable for $\mathrm{CMB}$ radiometric measurements, allowing differential measurements without the need of an active frontend switch.

In this scheme the best $1 / f$ noise suppression is reached when the radiometer is completely balanced. For slightly unbalanced configurations, e.g. when a cryogenic internal load is used as a reference signal, the effects introduced by the offset between the two inputs can be minimised after detection either by a variable DC gain stage that maintains the differenced output as close as possible to zero, or by multiplying in software one of the two signals by a factor, $r$, that must be properly tuned to balance the receiver. At first order the parameter $r$ is equal to the ratio between the sky and reference "total power" signal levels, i.e. $r \approx\left(\tilde{T}_{\text {sky }}+T_{\mathrm{n}}\right) /\left(\tilde{T}_{\text {ref }}+T_{\mathrm{n}}\right)$. With this value it is possible to obtain differenced noise streams with a knee frequency of the order of few tens of $\mathrm{mHz}$ and make the radiometer insensitive to fluctuations in the back-end amplifier gain.

The accuracy required in the calculation of $r$ from ground data must be determined by the instrument requirements on $1 / f$ noise and other systematic effects. In the case of PLANCK-LFI the main driver is the susceptibility to thermal fluctuations in the warm back-end stage, which can be maintained to required levels if $r$ is calculated with an accuracy ranging from $\pm 1 \%$ for the $30 \mathrm{GHz}$ channel to $\pm 0.3 \%$ for the $70 \mathrm{GHz}$ channel.

In our study we have considered three methods for calculating $r$, using total power radiometer data.

The most straightforward scheme uses the ratio of the average level of the total power signals. This method proves quite simple, accurate and relatively immune from systematic effects like $1 / f$ amplifier fluctuations, thermal effects etc. In order to be applied it is necessary that any spurious offset introduced by the back-end electronics is known with a relative accuracy of the order of $\pm 4 \%$. This is the baseline method in the case of PLANCK-LFI.

The second method uses the ratio of the standard deviations of the total power noise streams and is limited by its sensitivity to $1 / f$ fluctuations of the front-end amplifier noise temperature, which sets a lower limit on the expected accuracy that is about one order of magnitude worse than method 1.

The third method uses a minimisation strategy of the knee frequency of the differenced noise stream, by which it is possible to obtain the same level of accuracy of method 1, provided that the lenght of the total power data stream is enough to resolve the knee frequency of the differenced noise.

The application of this concept to prototype PLANCK-LFI radiometer data has shown that the "in software" offset balancing method is effective and that the balancing condition can be calculated from a limited amount of raw radiometric data in total power mode. In particular by methods 1 and 3 applied to a $15 \mathrm{~min}$ data stream of experimental radiometric data it was possible to calculate the same (at the level of $0.001 \%$ ) value of $r$.

Although in PLANCK-LFI method 1 is considered as baseline, the other methods will be used during the ground data analysis for cross-checks, as they are sensitive to non idealities in different ways.

Further studies will be aimed at a better understanding of the impact of systematic effects on the gain modulation factor accuracy and to continue the analysis of laboratory radiometer data in order to check our predictions. In addition we will continue with more realistic and detailed simulations.

Acknowledgements. The research described in this paper was performed in part at the Jet Propulsion Laboratory, California Institute of Technology, under a contract with the National Aeronautics and Space Administration.

\section{References}

Bennett, C., Bay, M., Halpern, M., et al. 2003, ApJ, 583, 1

Bersanelli, M., Maino, D., \& Mennella, A. 2002, Riv. Nuovo Cim., 25,1

Bersanelli, M., Mandolesi, N., \& Smoot, G. 1995, Mem. S.A.It., 66, 39

Janssen, M., D.Scott, White, M., et al. 1996, ArXiv Astrophysics e-prints [astro-ph/9602009]

Jarosik, N. 1996, IEEE Trans. Microwave Theory Tech., 44, 193

Jarosik, N., Bennett, C. L., Halpern, M., et al. 2003, ApJS, 145, 413

Lamarre, J.-M. 1997, in Microwave Background Anisotropies, ed. F. Bouchet, R. Gispert, B. Guilderdoni, \& J. Tran Thanh Van (Gif-sur-Yvette: Éditions Frontières), 31

Meinhold, P., Seiffert, M., Lubin, P., \& Childers, J. 1998, in II ESA Workshop on Millimeter Wave Technology and Applications, ed. J. Mallat, A. Raisanen, \& J. Tuovinen, 412

Mennella, A., Bersanelli, M., Burigana, C., et al. 2002, A\&A, 384, 736

Pospieszalski, M. W. 1989, IEEE Trans. Micro. Theory Techn., 37, 1340

Seiffert, M., Mennella, A., Burigana, C., et al. 2002, A\&A, 391, 1185

Smoot, G., Bennett, C., Weber, R., et al. 1990, ApJ, 360, 685

Smoot, G. F., Bennett, C. L., Kogut, A., et al. 1992, ApJL, 396, L1

Tuovinen, J., Bersanelli, M., \& Mandolesi, N. 2000, Astron. Astroph. Trans., 19, 551

Wollack, E. J. 1995, Rev. Scient. Instr., 66, 4305 